\title{
A Study of the Demographics of Web-Based Health-Related Social Media Users
}

Shouq A Sadah ${ }^{1}$, MS; Moloud Shahbazi ${ }^{1}$, MS; Matthew T Wiley ${ }^{1,2}$, MS; Vagelis Hristidis ${ }^{1}, \mathrm{PhD}$

${ }^{1}$ Department of Computer Science and Engineering, University of California, Riverside, Riverside, CA, United States

${ }^{2}$ SmartDocFinder LLC, Riverside, CA, United States

Corresponding Author:

Shouq A Sadah, MS

Department of Computer Science and Engineering

University of California, Riverside

351 Winston Chung Hall

900 University Ave

Riverside, CA, 92521

United States

Phone: 19517565691

Fax: 19518274643

Email: ssada003@cs.ucr.edu

\section{Abstract}

Background: The rapid spread of Web-based social media in recent years has impacted how patients share health-related information. However, little work has studied the demographics of these users.

Objective: Our aim was to study the demographics of users who participate in health-related Web-based social outlets to identify possible links to health care disparities.

Methods: We analyze and compare three different types of health-related social outlets: (1) general Web-based social networks, Twitter and Google+, (2) drug review websites, and (3) health Web forums. We focus on the following demographic attributes: age, gender, ethnicity, location, and writing level. We build and evaluate domain-specific classifiers to infer missing data where possible. The estimated demographic statistics are compared against various baselines, such as Internet and social networks usage of the population.

Results: We found that (1) drug review websites and health Web forums are dominated by female users, (2) the participants of health-related social outlets are generally older with the exception of the 65+ years bracket, (3) blacks are underrepresented in health-related social networks, (4) users in areas with better access to health care participate more in Web-based health-related social outlets, and (5) the writing level of users in health-related social outlets is significantly lower than the reading level of the population.

Conclusions: We identified interesting and actionable disparities in the participation of various demographic groups to various types of health-related social outlets. These disparities are significantly distinct from the disparities in Internet usage or general social outlets participation.

(J Med Internet Res 2015;17(8):e194) doi: 10.2196/jmir.4308

\section{KEYWORDS}

online social media; demographics; health forums; health care disparity; drug reviews

\section{Introduction}

\section{Background}

Social media have been employed in many industries to engage consumers. The health care industry has moved at a slower pace in incorporating social media because of inherent risks such as patient privacy, but recently this rate has increased to fulfill the consumers' needs [1]. Moreover, some companies use social media to provide their employees with wellness videos in order to cut their health care costs [2].

At the same time, health care disparity is a well-studied problem in which two population groups receive unequal services [3]. This problem has been analyzed across various dimensions relating to social determinants in health, including education 
and income, environmental hazards, and health outcomes such as mortality, morbidity, and behavioral risk factors [4]. However, health care disparity has not been studied in terms of social media participation. This is important as Internet access and participation in health communities has the potential to improve health outcomes [5]. Hence, understanding the demographics of social outlets, which is the focus of this paper, may shed light on another facet of health care disparity.

To cover different types of Web-based social outlets, we collected data from three types of sources: (1) general Web-based social networks, namely Google+ and Twitter, (2) drug review websites, and (3) health Web forums. We measure the following demographic attributes: age, gender, ethnicity, location, and writing level. Unfortunately, much of this information is unavailable for some, or all, of the sources. For that, we built and evaluated three classifiers for gender, ethnicity, and writing level. User names were used for the gender and ethnicity classifiers. Writing level for users was calculated using modified reading level formula to ignore very long incomprehensible sentences. To extract the location of a post, we use a geocoding application programming interface (API).

\section{Related Work}

\section{Analysis of Health-Related Social Outlets}

Many researchers have explored the effectiveness of Web-based social media in changing and improving the communication between providers and patients. According to Kane et al [6], 60 million Americans are using Health 2.0 applications, that is, social networks focused specifically on health care. Further, approximately $40 \%$ of Americans find an opinion in social media is more trustworthy if it conflicts with a professional's opinion or diagnosis. Hackworth and Kunz [7] found that $80 \%$ of American adults have looked online for health-related topics. Recently, there is increased interest in analyzing the health-related content of social media [1]. Denecke and Nejdl [8] analyzed medical concepts mentioned in medical social media posts from different sources to differentiate between informative and affective posts. They found that patients and nurses tend to share personal experiences, while physicians share health-related information. Lu et al [9] studied the content of three disease-specific health communities and their relationship to five informative topics: symptoms, complications, examination, drugs, and procedures. For example, users with breast cancer are more likely to discuss examination, while users with lung cancer are more likely to discuss symptoms. Wiley et al [10] analyzed the content of Web-based social media related to pharmaceutical drugs across several dimensions, including frequently mentioned diseases, keywords, and sentiment. While the aforementioned work examined health-related social media and content, none of them studied the demographics of the participating users, which is studied in this work.

\section{Measuring and Estimating Demographics of Users of Social Outlets}

\section{Survey-Based Methods}

In 2012, a Pew Internet Research study showed that women, age $30-49$, are more likely to participate in social media websites, where $75 \%$ of users are white [11]. eMarketer found that $68.9 \%$ of Hispanics use social media compared to $66.2 \%$ of the total population. Further, they showed that Hispanics are more likely to compare products online while shopping and write reviews on products [12]. However, no research has focused on health-related social media.

\section{Classifier-Based Methods}

Mislove et al [13] built methods to estimate both gender and ethnicity for Twitter users using the 1000 most popular first names reported by the US Social Security Administration and frequently occurring surnames reported by the 2000 US census. Gender and ethnicity methods used the reported first name and last name respectively. Mandel et al [14] analyzed the tweets related to Hurricane Irene using Mislove's gender classifier. We build on Mislove's work when creating our classifiers. While we also classify gender using first names, we extended these methods to screen names when a first name is not present. A related work for estimating reading levels of the US population [15] was presented to discuss limitations of low literacy patients. We measured the writing level based on this work since we did not encounter any similar work.

\section{Methods}

\section{Datasets}

Our analysis used data collected from three different types of health-related social outlets: general social networks, drug review websites, and health Web forums (Table 1 [16-23]). Google+ and Twitter were chosen as general social networks based on their popularity and number of users (we do not study Facebook because it offers no public interfaces to access its data). For drug review websites and health Web forums, three websites were selected for each, where we considered their breadth of topics and popularity. Figure 1 shows the overall process of our analysis, and Table 1 shows key statistics of each source including number of users, number of posts, and average sentence length. More information about the sources including start and end date is available in Multimedia Appendix 1. 
Table 1. Total number of users, posts, and average sentences length for each source.

\begin{tabular}{llll}
\hline Dataset & Users, $\mathrm{n}$ & Posts, $\mathrm{n}$ & Average sentence length (in words) \\
\hline TwitterHealth [16] & $5,095,849$ & $11,637,888$ & 10.82 \\
Google+Health [17] & 86,749 & 186,666 & 9.03 \\
Drugs.com [18] & 74,461 & 74,461 & 13.85 \\
DailyStrength/Treatments [19] & 213,524 & $1,055,603$ & 11.92 \\
WebMD/Drugs [20] & 122,040 & 122,040 & 13.53 \\
Drugs.com/Answers [21] & 201,126 & $5,948,877$ & 6.59 \\
DailyStrength/Forums [22] & 165,045 & $1,128,629$ & 13.2 \\
WebMD [23] & 155,912 & 320,118 & 15.37 \\
\hline
\end{tabular}

Table 2 shows which of the sources provide data for each of the five demographic attributes. Two demographic attributes are not present in any source: ethnicity and writing level. We therefore created methods to automatically classify these attributes, along with gender for the sources where unavailable. No indicates that the demographic attribute is not provided by the source. Yes indicates that the demographic attribute is provided by the source. Each classifier uses a distinct part of the user profile as denoted by the table footnotes. The writing level classifier uses the Flesch-Kincaid measure based on all users' posts [24].

Table 2. List of all used sources with the available attributes.

\begin{tabular}{|c|c|c|c|c|c|}
\hline Dataset & Age & Gender & Ethnicity & Location & Writing level \\
\hline TwitterHealth & No & Gender classifier $^{\mathrm{a}}$ & Ethnicity classifier $^{\mathrm{b}}$ & Yes & Writing level classifier \\
\hline Google+Health & Yes & Yes & & Yes & Writing level classifier \\
\hline Drugs.com & No & Gender classifier $^{\mathrm{c}}$ & No & No & Writing level classifier \\
\hline DailyStrength/Treatments & Yes & Yes & No & Yes & Writing level classifier \\
\hline WebMD/Drugs & Yes & Yes & No & No & Writing level classifier \\
\hline Drugs.com/Answers & No & Gender classifier $^{\mathrm{c}}$ & No & No & Writing level classifier \\
\hline DailyStrength/Forums & Yes & Yes & No & Yes & Writing level classifier \\
\hline WebMD & No & Gender classifier $^{\mathrm{c}}$ & No & No & Writing level classifier \\
\hline
\end{tabular}

${ }^{\mathrm{a}}$ First name.

${ }^{\mathrm{b}}$ Last name.

${ }^{\mathrm{c}}$ Screen name.

To filter health-related posts from Twitter and Google+, we built a list of 276 representative health-related keywords based on five categories:

1. Drugs: First we obtained a list of the 200 most popular drugs by prescriptions dispensed from RxList.com [25]. We then removed variants of the same drug (eg, different milligram dosages) resulting in 125 unique drug names.

2. Hashtags: We selected 11 popular health-related Twitter hashtags such as \#HCSM (Healthcare Communications \& Social Media).

3. Disorders: We selected 81 popular disorders such as cancer and Alzheimer.

4. Pharmaceuticals: We selected the 12 largest pharmaceutical companies such as Pfizer.

5. Insurance: We selected 44 of the biggest insurances such as Medicare and Humana. A complete list of used keywords can be found in Multimedia Appendix 1.

We used the Twitter streaming API [26], with these keywords as filters, to obtain the relevant tweets for our TwitterHealth dataset. Our Google+Health dataset was collected via the Google+ API [27], where each health-related keyword was used as a query to find relevant posts. For the drug review websites and health Web forums, we built custom crawlers in Java using the jsoup [28] library for crawling and parsing the hypertext markup language (HTML) content. For each source, we collected the available data, including user information, posts, disorder, or condition under which a discussion appears, keywords, tags, etc. We emphasize that we collected only publicly available data in accordance with each site's terms of use; no private data were collected. 
Figure 1. Overview of the data collection and analysis process.

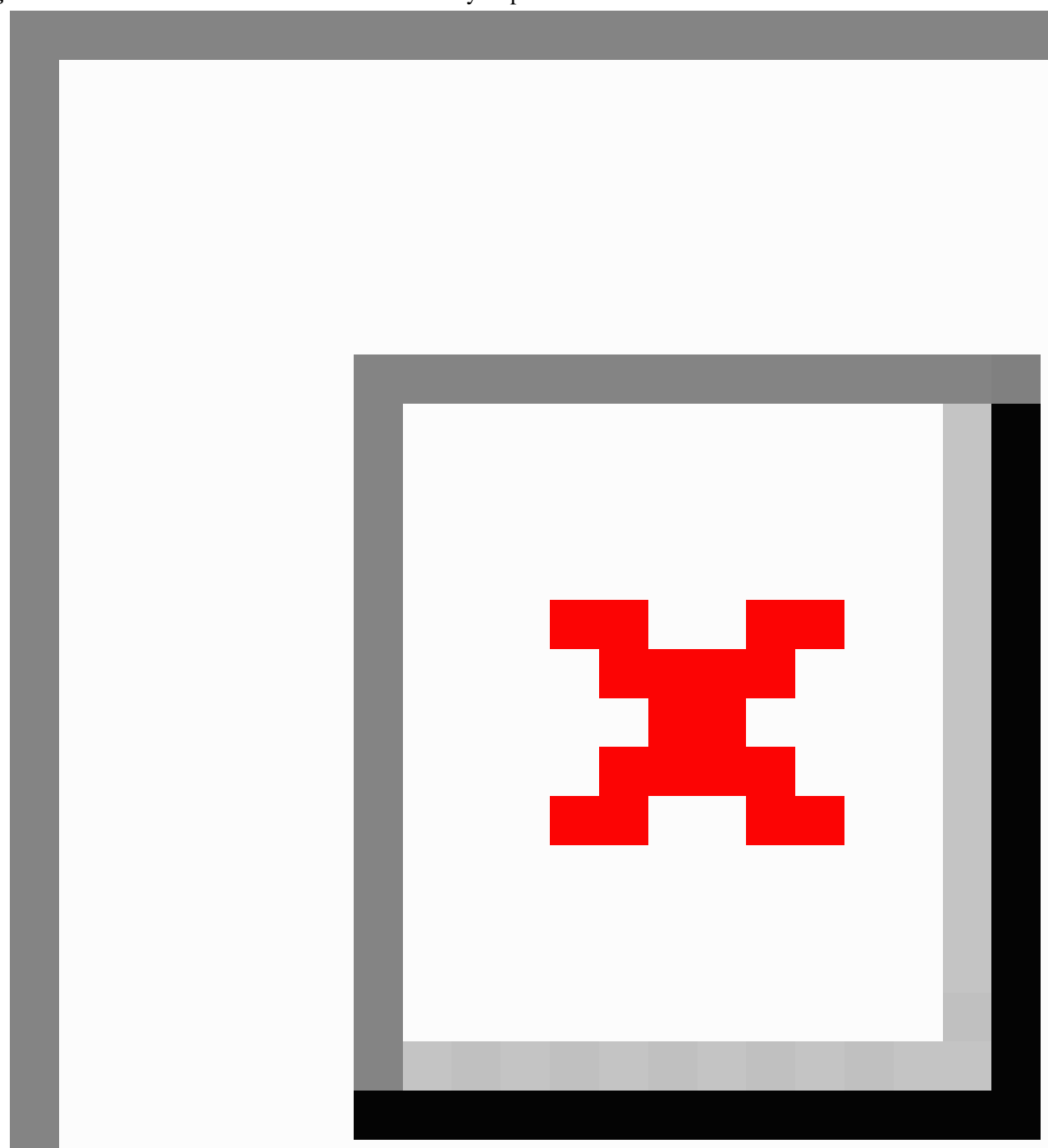

\section{User Demographics Estimation Methods}

\section{Overview}

We chose five demographic attributes as shown in Table 2: gender, age, ethnicity, location, and writing level. Since these attributes are not available in every source, we created several classifiers to derive missing attributes as specified in Table 2. Note that we do not fill missing values of users for sources that provide this information for at least some of their users, for example, if a user does not provide their age in Google+, we just ignore this user from the age-related analysis. Multimedia Appendix 1 shows the percentages of users who report each attribute in each source.

\section{Gender}

Four out of eight sources (Google+Health, DailyStrength/Treatments, WebMD/Drugs, and DailyStrength/Forums) allow users to report their gender (as shown in Table 2). Approximately $80 \%$ of the users of these sources chose to report it; thus, the reported gender was used for these sources.

For the other sources where gender is not available, we extended the methods of Mislove et al [13] to classify gender using the reported first name of users, if available; otherwise we extracted first names from user screen names. Note that screen names have not been used before, to the best of our knowledge, for gender estimation. In particular, we first collected the 1000 most 
popular male and female birth names reported by the US Social Security Administration [29] for each year from 1935 to 1995. Thus, we collected the names of people in 2014 aged 19-79 years old, which constitutes about $73.9 \%$ of the population [30]. There are 55,973 unique names in total. We further filtered this list to remove names with an aggregated frequency less than 10,000 or a discriminative gender probability less than $95 \%$. The resultant list contained 1328 names. For TwitterHealth and Google+Health, we checked if one of these 1328 first names is contained in the user-specified name to classify the user's gender. We first cleaned the first name by removing non-alphabetical characters and then performed case-insensitive string matching. Gender classifier evaluation is reported in Multimedia Appendix 1; the accuracy ranges from $76 \%$ to $99 \%$.

Age

Similarly, age was also reported in four sources (Google+Health, DailyStrength/Treatments, WebMD/Drugs, and DailyStrength/Forums).Three sources display the age as a single number, whereas one source displays age as a range (eg, 35-45). Approximately $61 \%$ of the users of these sources reported their age. When users provide an age range, the total number of users for each range is distributed uniformly to each year in the range. Ages are then grouped into five age groups: 0-17, 18-34, 35-44, 45-64, and 65 years and older. These age ranges are also used by the US census [31].

\section{Ethnicity}

The ethnicity of the users is not reported in any of the sources that we study; therefore, we created an ethnicity classifier similar to Mislove et al [13]. The 2000 US census, which is the most recent available, reports the distribution of ethnicities for each last name (last names with less than 100 individuals were omitted) [32]. For example, the distribution for Hernandez is reported as $4.55 \%$ white, $0.38 \%$ black, $0.27 \%$ Asian, and $93.81 \%$ Hispanic. We filtered this list to remove the last names with a frequency less than 1000 , or where the discriminative probability of the majority ethnicity is less than $80 \%$. We then use the ethnicity with the majority probability to classify ethnicity based on last name for sources that include the last name of users (Google+Health and TwitterHealth). We understand that race and ethnicity are not the same especially when referring to Hispanics, but in this paper we try to simplify the presentation by only reporting ethnicity, that is, we do not distinguish groups like white Hispanic versus black Hispanic, but only Hispanic. For the other sources (health Web forums and drug review websites), which do not have user names, we found that using the screen name for ethnicity estimation is inaccurate, and hence we do not report on the ethnicity of these sources. Ethnicity labeling and classifier evaluation is reported in Multimedia Appendix 1.

\section{Writing Level}

Different methods and formulas for measuring readability are available using different factors such as average number of syllables per words, average number of words per sentences, or average number of letters per words. In our work, we used the Flesch-Kincaid Grade Level [16] formula to estimate the writing level (values generally correspond to school grades 1-12) of the users:

Flesch-Kincaid Reading Age $=(0.39 \times$ ASL $)+(11.8 \times$ ASW $)$ - 15.59, where ASL is the Average Sentence Length, and ASW is the Average number of Syllables per Word.

Note that since we can only observe the text authored by users, we measure the writing level and not the reading level; however, we use the reading level formula since no alternative formula for the writing level exists. The writing level of a user is computed using the above equation by concatenating all of the user's posts and personal description. Links and hashtags from tweets are removed, and users with less than 100 words in total are ignored. We found that very high reading level was being assigned to users who write very long incomprehensible sentences. This is a case that was not considered by the original Flesch-Kincaid reading age formula, which assumed that the text is grammatically and syntactically correct (eg, the text of a novel). For that, we omit sentences with more than 30 words.

\section{Results}

\section{Overview}

To put our results in perspective, we compare them with other general demographics statistics. The population and Internet usage for each demographic group was obtained from the US census [29,30], while other statistics for Twitter and Google+ came from other sources [11,33-35]. Further, we compare the demographics of the users participating in health-related discussions on Twitter and Google+ to the overall demographics of the users in these sites. All our results are statistically significant, except the comparison between health Web forums and drug review websites with respect to gender and age group (0-17). Also, there is no significant difference between Google+Health and drug review websites for age group (35-44).

\section{Gender}

As shown in Table $3[11,30,31,33]$, the gender distribution in the population and Internet usage is almost the same, and there is a slight difference for general social networks. Our first key finding is that drug review websites and health Web forums are dominated by female users; the number of female users is almost four times larger than that of male users. TwitterHealth and Google+Health have similar gender ratios when compared to the overall user base of Twitter and Google+.

The finding that women use health forums much more than men is partially supported by previous research, which shows that women report ill health more frequently than men [36]. In contrast, this is not true for Twitter and Google+, which are dominated by news exchanges [37]. 
Table 3. Gender distribution for TwitterHealth, Google+Health, drug reviews, health forums, compared to other relevant populations.

\begin{tabular}{|c|c|c|}
\hline Source & Females, $\%$ & Males, $\%$ \\
\hline Population [30] & 51.05 & 48.95 \\
\hline Internet Use [31] & 51.63 & 48.37 \\
\hline General social networks [11] & 54.68 & 45.32 \\
\hline Twitter [33] & 57.00 & 43.00 \\
\hline Google+ [33] & 37.00 & 63.00 \\
\hline TwitterHealth $^{\mathrm{a}}$ & 51.81 & 48.19 \\
\hline Google + Health $^{\mathrm{a}}$ & 35.36 & 64.64 \\
\hline Drug review websites $^{\mathrm{a}}$ & 78.48 & 21.52 \\
\hline Health Web forums ${ }^{\mathrm{a}}$ & 78.41 & 21.59 \\
\hline
\end{tabular}

${ }^{\mathrm{a}}$ These results are from this work. Results in the rows above are reported in the respective citations.

\section{Age}

Table $4[30,31,34,38]$ reports the age distribution of users in the studied social outlets and in other relevant sources, to put the results in perspective. Age groups were chosen based on the US census. We therefore understand that the age ranges are not equal, but since our main goal is comparing the demographics of Web-based health-related social outlets to other statistics such as Internet usage, we chose to follow the census age ranges in computer and Internet access. Further, we provide population distribution in the Table 4 to compare each group size with others. One-fifth of Internet users are in the group 0-17; this percentage drops to approximately $1 \%$ for drug review websites and health Web forums. The majority of users on drug review websites are between 45 and 64 years old, and drug reviews have more users over 65 years than any other source; this is expected as older patients use more medications [39]. However, the percentage of drug review users above 65 is slightly lower than the percentage of Internet users over 65, which means that older people still have low participation in Health 2.0 sites. Also, the 18-34 age group dominates health Web forums, which is congruent with general social networks usage [34]. To summarize, our second key finding is that the participants of health-related social outlets are generally older than those of general-purpose social forums, but still relatively low in the $65+$ bracket. This is expected to change in the near future based on the participation statistics in the 45-64 bracket.

Table 4. Age distribution for Google+Health, drug reviews, health forums, and other relevant populations.

\begin{tabular}{llllll}
\hline Source & $0-17$ years, $\%$ & $18-34$ years, $\%$ & $35-44$ years, $\%$ & 45-64 years, \% & $65+$ years, $\%$ \\
\hline Population [30] & 24.00 & 23.11 & 12.93 & 26.53 & 13.44 \\
Internet use [31] & 19.30 & 27.55 & 14.99 & 28.36 & 9.80 \\
General social networks [34] & 14.58 & 27.43 & 20.68 & 30.98 & 6.32 \\
Google+ [38] & 8.08 & 71.61 & 11.08 & 7.82 & 1.42 \\
Google+Health $^{\mathrm{a}}$ & 3.42 & 53.21 & 21.89 & 19.02 & 2.46 \\
Drug review websites $^{\mathrm{a}}$ & 1.05 & 31.13 & 22.36 & 36.84 & 8.62 \\
Health Web forums $^{\mathrm{a}}$ & 1.03 & 39.80 & 25.81 & 28.95 & 4.41 \\
\hline
\end{tabular}

${ }^{\mathrm{a}}$ These results are from this work. Results in the rows above are reported in the respective citations.

\section{Ethnicity}

For the ethnicity and location analyses, we focus on the US population, in order to compare to available US census statistics. Table $5[12,31,40,41]$ shows the results of our ethnicity analysis.
Recall that users' ethnicity in Google+Health and TwitterHealth is classified using our last name-based classifier. Our third key observation is that blacks are underrepresented in health-related social network discussions (Google+Health, TwitterHealth). 
Table 5. Ethnicity distribution for TwitterHealth, Google+Health, and other relevant populations.

\begin{tabular}{|c|c|c|c|c|}
\hline Source & Asian, $\%$ & Black, $\%$ & Hispanic, $\%$ & White, \% \\
\hline Population [40] & 4.5 & 12.2 & 15.8 & 65.1 \\
\hline Internet use [31] & 5.5 & 11.7 & 13.9 & 67.2 \\
\hline General social networks [12] & 5.3 & 12.1 & 14.5 & 66.5 \\
\hline Twitter [41] & $\mathrm{N} / \mathrm{A}$ & 9 & 12 & 71 \\
\hline TwitterHealth $^{\mathrm{a}}$ & 3.24 & 0.3 & 23.5 & 73.0 \\
\hline Google + Health $^{\mathrm{a}}$ & 5.6 & 0.3 & 17.4 & 76.6 \\
\hline
\end{tabular}

${ }^{\mathrm{a}}$ These results are from this work. Results in the rows above are reported in the respective citations.

\section{Location}

Location is reported in four sources: the two general social networks (TwitterHealth, Google+Health), one drug review website (DailyStrength/Treatments), and one health Web forum (DailyStrength/Forums); approximately $62 \%$ of users reported their locations. For TwitterHealth and Google+Health, users report their location using a single string (eg, "NY, NY"). Thus, these strings are further processed to obtain structured locations (eg, state: New York, city: New York). In particular, non-alphanumeric characters and extra spaces were removed, and location strings with a frequency less than 14 were removed. This left us with about $60 \%$ of TwitterHealth and Google+Health users with location strings. Each location string was mapped to a location (city, state, country) using the Google Geocoding API [27]. We focus on US users, and hence we remove users from other countries. DailyStrength/Treatments and DailyStrength/Forums list the user's city and state separately; thus, we use the reported state for these sources.

In Figure 2, we show the distribution of users for each type of Web-based health-related social outlet, normalized by state population. Panel A shows the distribution of users in health
Web forums, Panel B shows the distribution of users in drug reviews websites, Panel $\mathrm{C}$ shows the distribution of users in TwitterHealth, and Panel D shows the distribution of users in Google+Health combined.

To better understand these results, we created Table 6 [31,42-45], which shows the correlation across all states between the normalized (by population) number of users in various health-related social outlets and other societal measures (see Multimedia Appendix 1 for more details). Our fourth key finding is that users in areas with higher income and more access to health care are more likely to participate in Web-based health-related outlets, and particularly in Web forums and drug review sites, which are the primary social sites for health-related information sharing [10]. Further, we see that in Twitter and Google+ the correlation with the number of physicians and education is higher. A reason could be that $59.1 \%$ of the 878,194 US active physicians [42] participate in these networks [46], which is a significant number, as the geolocated subsets of the Google+Health and TwitterHealth datasets contain only 882,207 users in the United States. The high correlation with education may be explained by the high percentage $(91 \%)$ of Twitter users with college degree or higher [47].

Table 6. Correlation across all states between the normalized (per capita) number of users for each type of health-related social outlets, and each state's population, normalized number of Internet users, normalized number of physicians, normalized number of uninsured patients, average annual income, and percentage of population with college degree or higher.

\begin{tabular}{|c|c|c|c|c|c|}
\hline Correlation & $\begin{array}{l}\text { Health Web } \\
\text { forums }\end{array}$ & $\begin{array}{l}\text { Drug review } \\
\text { websites }\end{array}$ & TwitterHealth & Google+Health & Google+ \\
\hline Internet usage [31] & 0.19 & 0.28 & 0.01 & -0.01 & 0.00 \\
\hline No. of physician [42] & 0.37 & 0.19 & 0.88 & 0.80 & 0.44 \\
\hline Uninsured population [43] & -0.40 & -0.40 & -0.17 & -0.11 & -0.10 \\
\hline Annual income [44] & 0.38 & 0.27 & 0.17 & 0.25 & 0.26 \\
\hline Education (ratio of people with a college degree) [45] & 0.35 & 0.22 & 0.56 & 0.63 & 0.54 \\
\hline
\end{tabular}


Figure 2. Per state capita number of users in (A) health web forums, (B) drug review websites, (C) TwitterHealth, and (D) Google+Health.

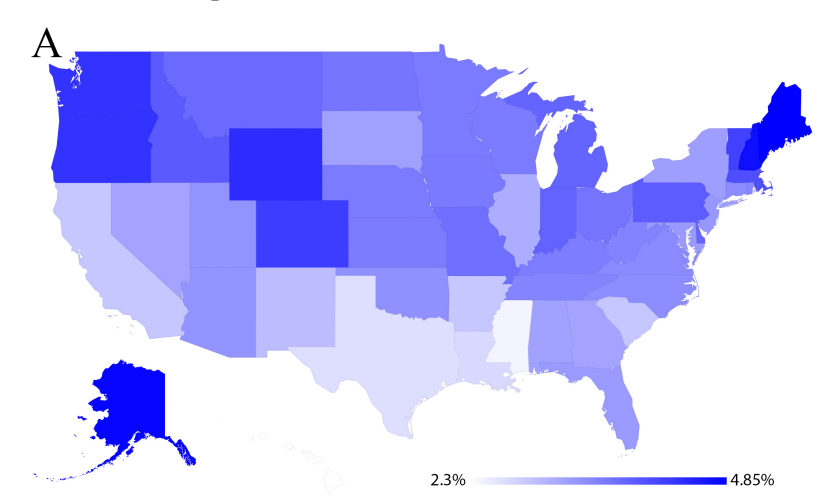

$\mathrm{C}$
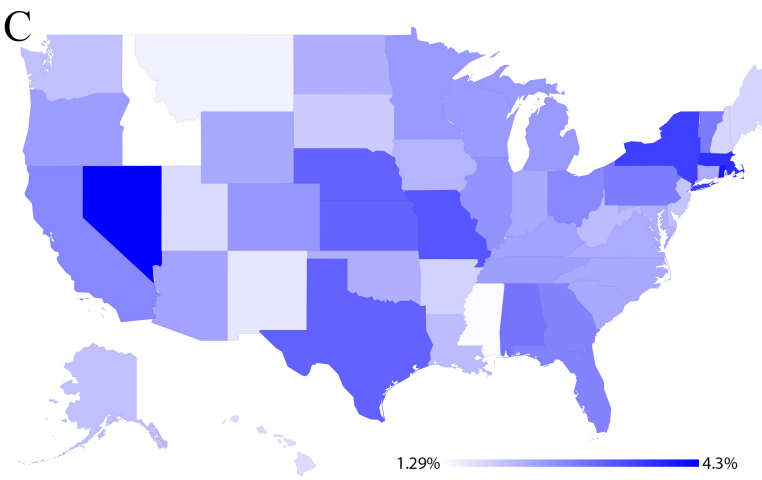

\section{Writing Level}

The writing level, as previously mentioned, is measured using a standard reading level formula that assigns a school grade to the given text. For example, when a person writes text at a $5^{\text {th }}$ grade reading level, it implies that their writing should be understood by people that have passed the $5^{\text {th }}$ grade. Table 7 reports our results for writing level of health-related social outlet
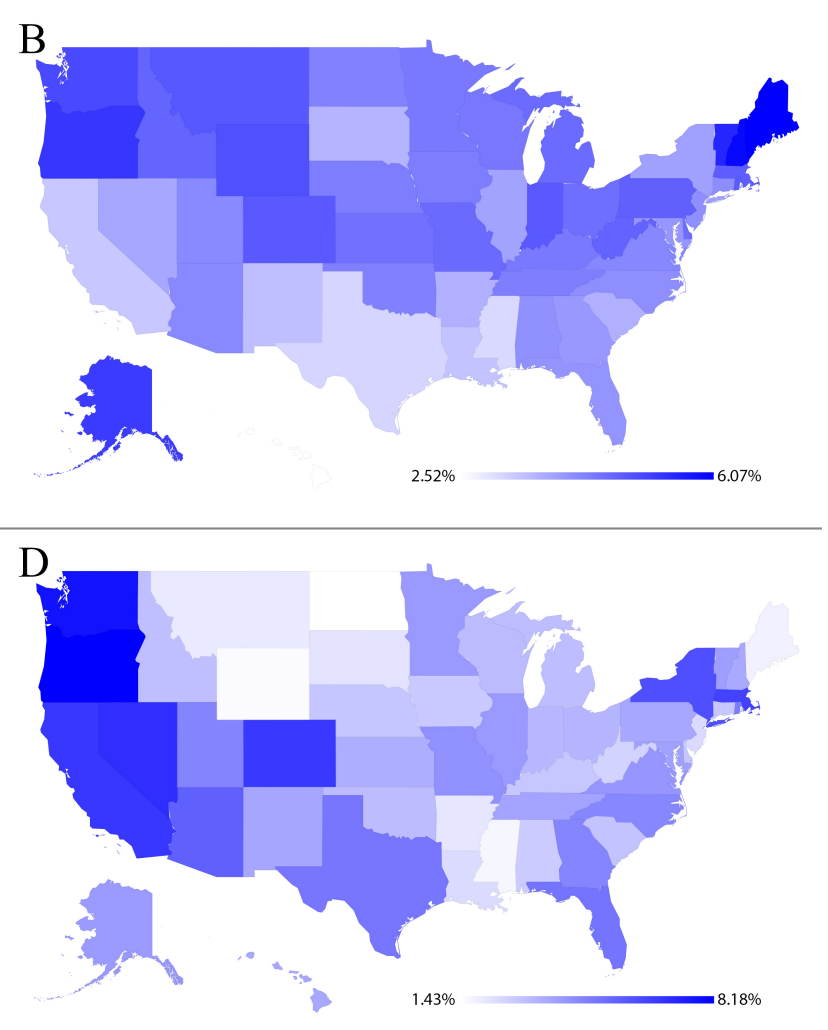

users. We see that Google+Health users have generally higher writing level than the rest sources, which may mean that more of the Google+Health users are professional accounts.

Next, we try to put these findings in perspective. Unfortunately, related work reports only on reading levels (and not writing levels) of the US population participating in social outlets. Thus, we compare our results in Table 7 to Figure 3, which reports the reading level of the general US population [15].

Table 7. Writing level distribution for TwitterHealth, Google+Health, drug reviews, and health forums.

\begin{tabular}{|c|c|c|c|}
\hline Source & Age $0-5, \%$ & Age $6-9, \%$ & Age $10-16, \%$ \\
\hline TwitterHealth & 37.77 & 51.09 & 11.13 \\
\hline Google+Health & 6.45 & 55.63 & 37.91 \\
\hline Drug review websites & 30.42 & 66.17 & 3.41 \\
\hline Health Web forums & 28.79 & 68.24 & 2.98 \\
\hline
\end{tabular}

Our fifth key finding is that the writing level in health-related social outlets (Table 7) is generally lower than the reading level of the population (Figure 3). Thus users/patients can easily comprehend the posts and hence benefit from the experiences of other users. The benefit of social interaction with respect to health empowerment has been demonstrated before [48]. In an online epilepsy community, 59\% of patients found another patient experiencing the same symptoms, $58 \%$ had a better understanding of seizures, and 55\% learned more about treatments and symptoms. 
Figure 3. Reading level of US population.

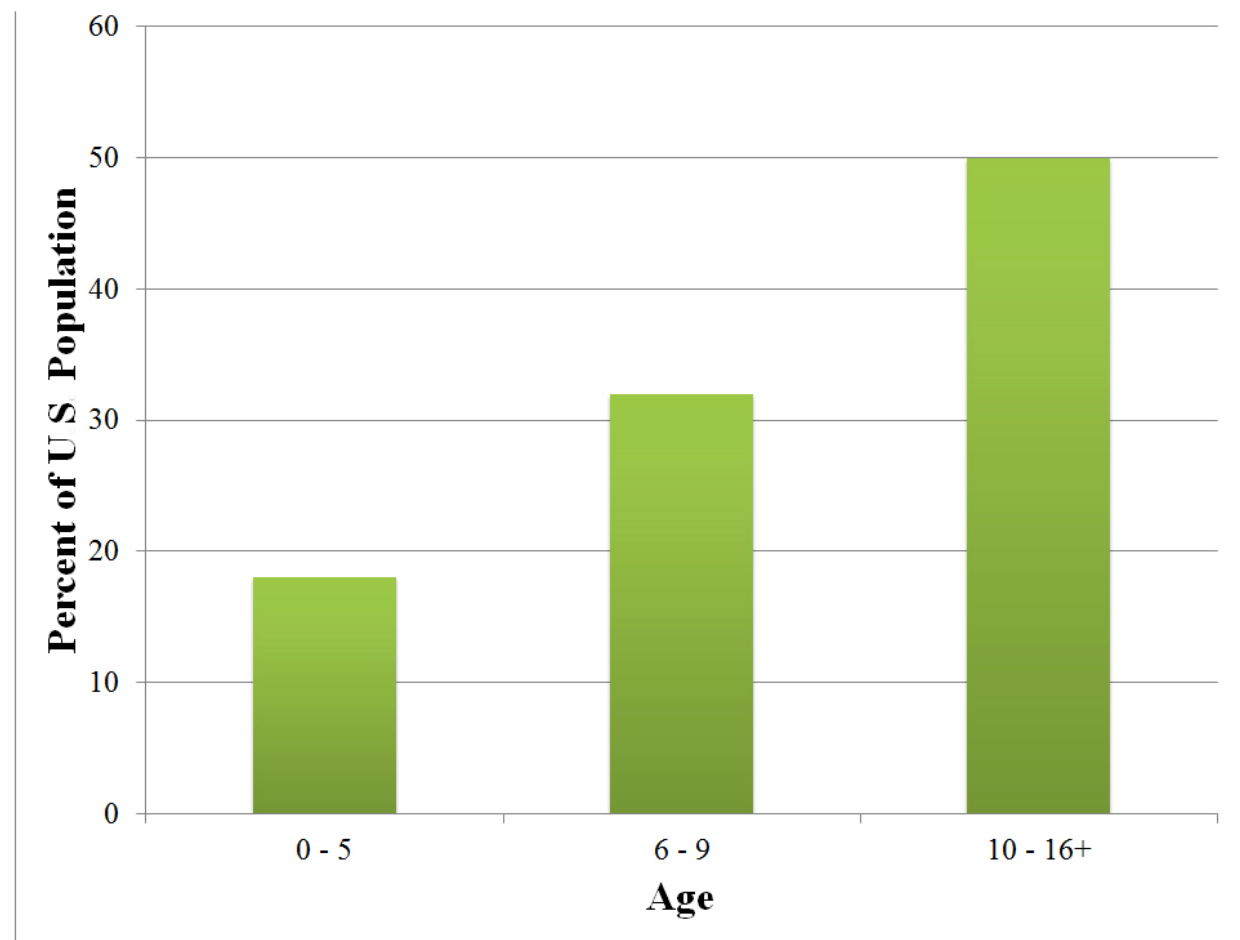

\section{Statistical Significance Tests}

Tables 8 and 9 report the $P$ values for Pearson's chi-square test of independence and the Mann-Whitney U test. Note that we compute only significance values between sources that we have analyzed and not between our sources and sources analyzed by other works (such as Google+ [38]), since we do not have the raw data for those sources.

Table 8. $P$ values for Pearson's chi-square test of independence.

\begin{tabular}{llll}
\hline & Gender & Age & Ethnicity \\
\hline TwitterHealth vs Google+Health & $<.001$ & N/A & $<.001$ \\
TwitterHealth vs Health Web forums & $<.001$ & N/A & $<.001$ \\
TwitterHealth vs Drug review websites & $<.001$ & N/A & $<.001$ \\
Google+Health vs Health Web forums & $<.001$ & $<.001$ & $<.001$ \\
Google+Health vs Drug review websites & $<.001$ & $<.001$ & $<.001$ \\
Health Web forums vs Drug review websites & $<.001$ & $<.001$ & $<.001<$ \\
\hline
\end{tabular}


Table 9. $P$ values for Mann-Whitney $\mathrm{U}$ test.

\begin{tabular}{|c|c|c|c|c|c|c|}
\hline & $\begin{array}{l}\text { TwitterHealth vs } \\
\text { Google+Health }\end{array}$ & $\begin{array}{l}\text { TwitterHealth vs } \\
\text { Health Web forums }\end{array}$ & $\begin{array}{l}\text { TwitterHealth vs } \\
\text { Drug review web- } \\
\text { sites }\end{array}$ & $\begin{array}{l}\text { Google+Health vs } \\
\text { Health Web forums }\end{array}$ & $\begin{array}{l}\text { Google+Health vs } \\
\text { Drug review websites }\end{array}$ & $\begin{array}{l}\text { Health Web fo- } \\
\text { rums vs Drug } \\
\text { review websites }\end{array}$ \\
\hline \multicolumn{7}{|l|}{ Gender } \\
\hline Male & $<.001$ & $<.001$ & $<.001$ & $<.001$ & $<.001$ & .5797 \\
\hline Female & $<.001$ & $<.001$ & $<.001$ & $<.001$ & $<.001$ & .5797 \\
\hline \multicolumn{7}{|l|}{ Age } \\
\hline $0-17$ & N/A & N/A & N/A & $<.001$ & $<.001$ & .5144 \\
\hline $18-34$ & N/A & N/A & N/A & $<.001$ & $<.001$ & $<.001$ \\
\hline $35-44$ & N/A & N/A & N/A & .01661 & .7747 & $<.001$ \\
\hline $45-64$ & N/A & N/A & N/A & $<.001$ & $<.001$ & $<.001$ \\
\hline$\geq 65$ & N/A & N/A & N/A & .01066 & $<.001$ & $<.001$ \\
\hline \multicolumn{7}{|l|}{ Ethnicity } \\
\hline White & $<.001$ & $<.001$ & $<.001$ & $<.001$ & $<.001$ & .1316 \\
\hline Black & .6339 & $<.001$ & $<.001$ & $<.001$ & $<.001$ & .0944 \\
\hline Asian & $<.001$ & $<.001$ & $<.01$ & $<.001$ & $<.001$ & .8054 \\
\hline Hispanic & $<.001$ & $<.001$ & $<.001$ & $<.001$ & $<.001$ & .6503 \\
\hline \multicolumn{7}{|c|}{ Writing level } \\
\hline $0-5$ & $<.001$ & $<.001$ & $<.001$ & $<.001$ & $<.001$ & $<.001$ \\
\hline $6-9$ & $<.001$ & $<.001$ & $<.001$ & $<.001$ & $<.001$ & $<.001$ \\
\hline $10-16$ & $<.001$ & $<.001$ & $<.001$ & $<.001$ & $<.001$ & .00516 \\
\hline
\end{tabular}

\section{Discussion}

\section{Principal Findings}

Our results can help health care providers customize educational campaigns for different groups. For example, white women should be informed to a larger extent on the possible misinformation spreading in health Web forums, since they participate much more.

Regarding mitigating ethnicity-based health care disparities, we found that Twitter and Google+ are more effective in reaching out to Hispanics about health care offerings. However, this is not true for black ethnicity, who are not overrepresented in any health-related social outlet. This means that there is no single outlet to reach black population, which has been shown to receive worse health care by about $40 \%$ comparing to white population [49].

Advertisers may use our results to decide on the best sites to advertise their products; for instance, drug review websites are more appropriate than Google+ to advertise drugs for the 45-64 age bracket, but the opposite is true for the 18-34 age bracket. Further, drug review websites and health Web forums are better to target females when advertising for their products than other health-related social outlets.

In the age results section, we found that younger groups (18-34 years old) participate in large numbers in health forums, which may sound counterintuitive. By analyzing posts for this age bracket, we found the most popular keywords are related to pregnancy such as birth control, ovulation, and miscarriage. On the other hand, their participation is lower for drug review websites. A possible explanation may be that often patients who talk about pregnancy are not taking any drugs, compared to other conditions like diabetes, where drugs are more common.

We also attempt to explain the disparities in the participation in health-related social outlets based on socioeconomic factors through the state-level participation distributions. Our results in Table 6 show that less access to physicians does not lead to higher participation in health-related social outlets as one would expect. In contrast, it seems that the participation in such outlets is correlated with the access to health care and the average income.

The weak but positive correlation between income and participation to health Web forums and drug review sites may be partially attributed to the higher Internet usage of the more affluent groups, as shown in Table 6. Another possible explanation is that lower income or uninsured persons are more likely to be part of a community with health care disparities [50].The positive correlation between education and participation in health-related social outlets, especially Google+Health and TwitterHealth, may be partially explained by the fact that people with college degrees are less likely to be uninsured, since $10 \%$ of college graduates are uninsured, compared to $40 \%$ of adults who have not graduated from high school [51]. In addition, $60 \%$ of uninsured people are from families with low incomes [51], and the group of people with income lower than US $30 \mathrm{~K}$ is the lowest group in terms of accessing health information [52], Hence, our results show that people with low income have less access to health information. 
On the other hand, we found that the content in health-related social outlets is easy to understand for almost all users, given the low writing level. That is, the well-known health literacy issue, which is more severe in low-income and lower education populations [5], does not seem to apply to Web-based health-related social outlets. Of course, the low writing level does not address the issue of language, as many low income and low education users in the United States do not speak English at home [53].

\section{Limitations}

Our ethnicity and gender classifiers are not perfect, as shown in Multimedia Appendix 1, and thus introduce an error into our analyses. This issue is less significant for gender, since out of all users included in our gender analysis for health Web forums and drug review websites, a majority of the users (over 94\%) report their gender, and hence the classifier was only used for $6 \%$ of users. Further, a majority of users in drug review websites and health Web forums are female, and our gender classifier obtained an accuracy greater than $99 \%$ for females when using a screen name.

Another limitation is the informal writing style of social media posts, as our writing level method uses the average sentence length, which expects that posts are properly punctuated. We addressed this limitation to some degree by only considering sentences of a reasonable length (less than 30 words). Estimating writing level could have been improved by considering other features like typos or spelling mistakes. Further, it would be useful to measure the quality of the posted information, in addition to just the writing level. This is a challenging issue, which we leave as future work.

Since all the attributes are reported by users, there is inevitably self-selection bias. In particular, gender, age, and location are not mandatory in any site. For instance, older people may choose not to report their age. Moreover, choosing to report the real names or posting profile pictures could also create self-selection bias in our gender and ethnicity classifiers. There may also be various types or degrees of bias across different outlets. For instance, WebMD users may use their real name less frequently than Twitter users. This in turn may bias the study results, especially for ethnicity where we depend completely on the classifier results.

\section{Conclusion}

We studied user demographics in Web-based health-related social outlets, which we split into three different types: social networks, drug review websites, and health Web forums. The distributions of the demographic attributes-gender, age, ethnicity, location, and writing level—have been analyzed for each source type and compared with relevant baseline user distributions like Internet and general social outlets participation. The results reveal interesting and often unexpected disparities with respect to all demographic attributes.

\section{Acknowledgments}

This project was partially supported by National Science Foundation (NSF) grants IIS-1216007, IIS-1447826, and IIP-1448848. Any opinions, findings and conclusions or recommendations expressed in this material are those of the authors and do not necessarily reflect the views of the National Science Foundation (NSF).

\section{Authors' Contributions}

All authors contributed substantially to this work. They designed and performed the analysis and approved the final version of this manuscript.

\section{Conflicts of Interest}

None declared.

\section{Multimedia Appendix 1}

Online social outlets summary, health keywords, classifiers evaluation, and data coverage.

\section{[PDF File (Adobe PDF File), 36KB-Multimedia Appendix 1]}

\section{References}

1. ECRI. 2011. Social media in healthcare URL: http://kcmsociety.org/wp-content/uploads/2013/04/ECRI-social-media.pdf [accessed 2015-02-02] [WebCite Cache ID 6W1rk2Yt2]

2. Giving benefits the YouTube treatment - workplace benefits association. 2008. URL: http://www.workplacebenefits.org/ news/giving-benefits-youtube-treatment-711171-1.html [accessed 2015-01-31] [WebCite Cache ID 6Vyr2D7tA]

3. Health Disparities. URL: http://www.medicaid.gov/Medicaid-CHIP-Program-Information/By-Topics/Data-and-Systems/ Health-Disparities.html[WebCite Cache ID 6W1rr2Maq]

4. Frieden TR, Centers for Disease Control and Prevention (CDC). Forward: CDC Health Disparities and Inequalities Report - United States, 2011. MMWR Surveill Summ 2011 Jan 14;60 Suppl:1-2 [FREE Full text] [Medline: 21430612]

5. Gibbons MC, Fleisher L, Slamon RE, Bass S, Kandadai V, Beck JR. Exploring the potential of Web 2.0 to address health disparities. J Health Commun 2011;16 Suppl 1:77-89. [doi: 10.1080/10810730.2011.596916] [Medline: 21843097] 
6. Kane GC, Fichman RG, Gallaugher J, Glaser J. Community relations 2.0. Harv Bus Rev 2009 Nov;87(11):45-50, 132. [Medline: 19891388]

7. Hackworth B, Kunz M. Health care and social media: building relationships via social networks. Acad Heal Care J 2010;6(1):55-69 [FREE Full text]

8. Denecke K, Nejdl W. How valuable is medical social media data? Content analysis of the medical web. Information Sciences 2009 May 30;179(12):1870-1880. [doi: 10.1016/j.ins.2009.01.025]

9. Lu Y, Zhang P, Liu J, Li J, Deng S. Health-related hot topic detection in online communities using text clustering. PLoS One 2013;8(2):e56221 [FREE Full text] [doi: 10.1371/journal.pone.0056221] [Medline: 23457530]

10. Wiley MT, Jin C, Hristidis V, Esterling KM. Pharmaceutical drugs chatter on Online Social Networks. J Biomed Inform 2014 Jun;49:245-254 [FREE Full text] [doi: 10.1016/j.jbi.2014.03.006] [Medline: 24637141]

11. Pew Research Center's Internet \& American Life Project. Social Networking Fact Sheet URL: http://www.pewinternet.org/ fact-sheets/social-networking-fact-sheet/[WebCite Cache ID 6W22L3O2w]

12. eMarketer. 2013. Health care and social media: bulding relationships via social networks URL: http://www.emarketer.com/ Article/Hispanic-Shoppers-Bring-Social-Mobile-Habits-Grocery-Aisle/1009839 [accessed 2015-02-02] [WebCite Cache ID 6W21kTHSr]

13. Mislove A, Lehmann S, Ahn Y. AAAI. 2011. Understanding the demographics of Twitter users URL: http://www.ccs.neu.edu/ home/amislove/publications/Twitter-ICWSM.pdf [accessed 2015-06-14] [WebCite Cache ID 6ZHoYQIfV]

14. Mandel B, Culotta A, Boulahanis J. A demographic analysis of online sentiment during hurricane irene. Language in Social Media 2012:27-36 [FREE Full text]

15. Harvard. The literacy problem. URL: http://cdn1.sph.harvard.edu/wp-content/uploads/sites/135/2012/09/doakchap1-4.pdf [accessed 2015-06-14] [WebCite Cache ID 6ZHoudAjI]

16. Twitter, Inc. URL: https://twitter.com/ [accessed 2015-08-01] [WebCite Cache ID 6aTENJsel]

17. Google+. URL: https://accounts.google.com/ServiceLogin?service=oz\&passive=1209600\&continue=https://plus.google.com/ ?gpsrc\%3Dgplp0 [accessed 2015-08-01] [WebCite Cache ID 6aTEQOvFn]

18. Drugs.com. Prescription Drug Information, Interactions \& Side Effects URL: http://www.drugs.com/ [accessed 2015-02-02] [WebCite Cache ID 6W231Hwlt]

19. DailyStrength. Treatments: reviews of drugs, therapies and remedies by everyday people URL: http://www.dailystrength.org/ treatments [accessed 2015-02-02] [WebCite Cache ID 6W23nXoJa]

20. WebMD Drugs \& Treatments - Medical Information and user ratings on prescription drugs and over-the-counter (OTC) medications. URL: http://www.webmd.com/drugs/index-drugs.aspx [accessed 2015-02-02] [WebCite Cache ID 6W23rKUfB]

21. Drugs.com. Medical Questions Answered URL: http://www.drugs.com/answers/ [accessed 2015-02-02] [WebCite Cache ID 6W23vDAi5]

22. DailyStrength. Online Support Groups URL: http://www.dailystrength.org/support-groups [accessed 2015-02-02] [WebCite Cache ID 6W23ztpRe]

23. WebMD - Better information. Better health URL: http://www.webmd.com/ [accessed 2015-02-02] [WebCite Cache ID 6W247u0HQ]

24. The Flesch grade level readability formula. URL: http://www.readabilityformulas.com/flesch-grade-level-readability-formula. php [accessed 2015-02-02] [WebCite Cache ID 6W22mEB3h]

25. RxList - The Internet Drug Index for prescription drugs, medications and pill. URL: http://www.rxlist.com/script/main/hp. asp [accessed 2015-02-02] [WebCite Cache ID 6W24Layfl]

26. The Streaming APIs | Twitter Developers. URL: https://dev.twitter.com/streaming/overview [accessed 2015-06-14] [WebCite Cache ID 6ZHpD7gjO]

27. Google+ API - Google+ Platform - Google Developers. URL: https://developers.google.com/+/api/ [accessed 2015-02-02] [WebCite Cache ID 6W24x2YYc]

28. jsoup Java HTML Parser, with best of DOM, CSS, and jquery. URL: http://jsoup.org/ [accessed 2015-02-02] [WebCite Cache ID 6W2528eFu]

29. Top names over the last 100 years. URL: http://www.ssa.gov/oact/babynames/decades/century.html [accessed 2015-02-02] [WebCite Cache ID 6W25cpwBx]

30. U.S. Census Bureau Demographic Internet Staff. 2012. Age and sex composition in the United States URL: https://www. census.gov/population/age/data/2012comp.html [accessed 2015-02-02] [WebCite Cache ID 6W25iCZh0]

31. U.S. Census Bureau Demographic Internet Staff. 2012. Computer and internet access in the United States URL: https:/ /www.census.gov/hhes/computer/publications/2012.html [accessed 2015-02-02] [WebCite Cache ID 6W271RtOB]

32. US Census Bureau Data Integration Division. Genealogy Data - Frequently occurring surnames from Census 2000 - US Census Bureau URL: http://www.census.gov/topics/population/genealogy/data/2000 surnames.html [accessed 2015-02-02] [WebCite Cache ID 6W27P3i6N]

33. Smith C. User demographics of the major social networks. 2012. URL: http://expandedramblings.com/index.php/ user-demographics-of-the-major-social-networks-infographic/ [accessed 2015-02-02] [WebCite Cache ID 6W27uwrNE]

34. April 2011: The Days of Double-Digit Growth in Social Network Users Are Over. URL: http://www.iab.net/research/ industry data and landscape/1675/1644724 [accessed 2015-02-02] [WebCite Cache ID 6W284mBJz] 
35. Finn G. Behind The Numbers Of Google+'s Monumental Rise To 25 Million Visitors. 2011. URL: http://searchengineland. com/behind-the-numbers-of-googles-monumental-rise-to-25-million-unique-visitor-88076 [accessed 2015-02-02] [WebCite Cache ID 6W28agd5M]

36. Ashley J. BBC News - Women more likely to report ill health than men. 2010. URL: http://news.bbc.co.uk/2/hi/health/ 8588686.stm [accessed 2015-02-02] [WebCite Cache ID 6W28nDUGK]

37. Kwak H, Lee C, Park H, Moon S. What is Twitter, a Social Network or a News Media? ACM 2010 Apr:591-600 [FREE Full text]

38. The comprehensive resource for Google+ trends and statistics. URL: http://www.gplusdata.com/users/sign in [accessed 2015-02-02] [WebCite Cache ID 6W29H2CHP]

39. Gu Q, Dillon CF, Burt VL. Prescription drug use continues to increase: U.S. prescription drug data for 2007-2008. NCHS Data Brief 2010 Sep(42):1-8 [FREE Full text] [Medline: 20854747]

40. US Census Bureau. 2012. Statistical abstract of the United States - Section 1 URL: http://www.census.gov/prod/2011pubs/ 12statab/pop.pdf [accessed 2015-02-01] [WebCite Cache ID 6W29OSGmX]

41. Bennett S. Twitter, Facebook and LinkedIn: age, ethnicity and gender of the major social networks. 2011. URL: http://www. adweek.com/socialtimes/pew-social-network-age-ethnicity-gender/453165?red=at [accessed 2015-02-02] [WebCite Cache ID 6W2A812Cu]

42. Young A, Chaudhry H, Thomas JV, Dugan M. A census of actively licensed physicians in the United States. J Med Regul 2012;99:11-24 [FREE Full text]

43. Denavas-walt B, Proctor B, Smith J. US Census Bureau, Current Population Reports. Washington, DC; 2013. Income, poverty, and health insurance coverage in the United States URL: http://www.census.gov/prod/2013pubs/p60-245.pdf [accessed 2015-02-02] [WebCite Cache ID 6W2D1eglk]

44. US Census Bureau Data Integration Division. Income URL: http://www.census.gov/hhes/www/income/data/statemedian/ [accessed 2015-02-02] [WebCite Cache ID 6W2D9aO6n]

45. US Census Bureau. 2012. Statistical abstract of the United States - Section 4 URL: http://www.census.gov/prod/2011pubs/ 12statab/educ.pdf [accessed 2015-02-02] [WebCite Cache ID 6W2DHYzQJ]

46. Cooper CP, Gelb CA, Rim SH, Hawkins NA, Rodriguez JL, Polonec L. Physicians who use social media and other internet-based communication technologies. J Am Med Inform Assoc 2012;19(6):960-964 [FREE Full text] [doi: 10.1136/amiajnl-2011-000628] [Medline: 22634078]

47. Hampton KN, Goulet LS, Rainie L, Purcell K. Pew Research Center's Internet \& American Life Project. 2011 Jun 16. Social networking sites and our lives URL: http://www.pewinternet.org/2011/06/16/social-networking-sites-and-our-lives/ [accessed 2015-08-01] [WebCite Cache ID 6aTEsGHIL]

48. Wicks P, Keininger DL, Massagli MP, de la Loge C, Brownstein C, Isojärvi J, et al. Perceived benefits of sharing health data between people with epilepsy on an online platform. Epilepsy Behav 2012 Jan;23(1):16-23 [FREE Full text] [doi: 10.1016/j.yebeh.2011.09.026] [Medline: 22099528]

49. Agency for Healthcare Research \& Quality (AHRQ). 2014. Disparities in healthcare quality among racial and ethnic minority groups URL: http://www.ahrq.gov/research/findings/nhqrdr/nhqrdr10/minority.html [accessed 2015-06-14] [WebCite Cache ID 6ZHq5iyFV]

50. Mendes E. Gallup poll. 2010. In US, health disparities across incomes are wide-ranging URL: http://www.gallup.com/poll/ 143696/health-disparities-across-incomes-wide-ranging.aspx [accessed 2015-02-02] [WebCite Cache ID 6W2E9gDMj]

51. Adler N, Newman K. Socioeconomic Disparities In Health: Pathways And Policies. Health Affairs 2002 Mar 01;21(2):60-76 [FREE Full text] [doi: 10.1377/hlthaff.21.2.60]

52. Madden M. Pew Research Center. 2013. Technology use by different income groups URL: http://www.pewinternet.org/ 2013/05/29/technology-use-by-different-income-groups/ [accessed 2015-06-14] [WebCite Cache ID 6ZHs8GsTY]

53. Ryan C. US Census Bureau. 2013. Language Use in the United States URL: http://www.census.gov/prod/2013pubs/acs-22. pdf [accessed 2015-02-01] [WebCite Cache ID 6W2EHGxIj]

\section{Abbreviations}

API: application programming interface 
Edited by G Eysenbach; submitted 02.02.15; peer-reviewed by G Luo, M Tremblay; comments to author 12.03.15; revised version received 15.06.15; accepted 03.07.15; published 06.08.15

Please cite as:

Sadah SA, Shahbazi M, Wiley MT, Hristidis V

A Study of the Demographics of Web-Based Health-Related Social Media Users

$J$ Med Internet Res 2015;17(8):e194

URL: http://www.jmir.org/2015/8/e194/

doi: 10.2196/jmir.4308

PMID: 26250986

(C) Shouq A Sadah, Moloud Shahbazi, Matthew T Wiley, Vagelis Hristidis. Originally published in the Journal of Medical Internet Research (http://www.jmir.org), 06.08.2015. This is an open-access article distributed under the terms of the Creative Commons Attribution License (http://creativecommons.org/licenses/by/2.0/), which permits unrestricted use, distribution, and reproduction in any medium, provided the original work, first published in the Journal of Medical Internet Research, is properly cited. The complete bibliographic information, a link to the original publication on http://www.jmir.org/, as well as this copyright and license information must be included. 